\title{
Current Clinical Methods for Detection of Peri-Prosthetic Joint Infection
}

\author{
Ellis Berns, ${ }^{2}$ Caitlin Barrett, ${ }^{1}$ Mursal Gardezi, ${ }^{1}$ Carole Spake, ${ }^{2}$ Jillian Glasser, ${ }^{1}$ \\ Valentin Antoci, ${ }^{2,3}$ Christopher T. Born, ${ }^{2,3}$ and Dioscaris R. Garcia ${ }^{1-3}$
}

\begin{abstract}
Background: Currently, one of the most pressing problems in the field of orthopedic surgery is peri-prosthetic joint infection [PJI]. While there are numerous ways to detect PJI, current clinical detection methods differ across institutions and have varying criteria and protocols. Some of these methods include the Modified Musculoskeletal Infection Society system, culturing, polymerase chain reaction, the determination of the presence of certain biomarkers, testing for the presence of alpha defensin peptides, and leukocyte level testing.

Methods: This review summarizes the most recent publications in the field of PJI detection to highlight current strengths as well as provide future directions to find the system for the quickest, cost-effective, and most accurate way to diagnose these types of infections.

Results: The results of this literature review suggest that, while each method of diagnosis has its advantages, each has various drawbacks as well. Current methods can be expensive, take days to weeks to complete, be prone to contamination, and can produce ambiguous results.

Conclusions: The findings in this review emphasize the need for a more comprehensive and accurate system for diagnosing PJI. In addition, the specific comparison of advantages and drawbacks can be useful for researchers and clinicians with goals of creating new diagnostic tests for PJIs, as well as in clinical scenarios to determine the correct treatment for patients.
\end{abstract}

Keywords: implant; orthopedic infection; peri-prosthetic joint infection

$\mathbf{T}$ HE DIFFICULTY IN ESTABLISHING a diagnosis of periprosthetic joint infection [PJI] continues to impact adversely the field of orthopedics. The burden of misdiagnosis impedes optimal patient care and physician decision making [1]. The PJI significantly decreases patient quality of life [2] and places an economic burden on the healthcare system [3].

The current gold standard for diagnosis of PJI is the Modified Musculoskeletal Infection Society (MSIS) criteria [4-7]. The MSIS considers major and minor criteria. Infections meeting the major MSIS criteria may include two positive cultures of phenotypically identical organisms or the presence of a sinus tract, but the criteria can fail to detect borderline infections that meet fewer than three minor criteria $[7,8]$. Infections with low virulence organisms complicate PJI diagnosis because patients can present with reduced clinical symptoms and normal conventional laboratory values [9].
While the MSIS has led to a procedural standardization for PJI detection across hospital systems [10], the need for novel tests with high sensitivity and specificity to aid in PJI diagnosis remains pressing. An ideal detection method should be fast, accurate, specific, easy to use, inexpensive, and have a low rate of contamination. This review will summarize MSIS and the current clinical methods of microbial detection for diagnosis of PJI.

\section{Methods}

MSIS

Although other rating systems exist, MSIS is the gold standard for the definitive diagnosis of PJI and the evaluation of new tests. The two major MSIS criteria require direct visu-

\footnotetext{
${ }^{1}$ Brown University, Providence, Rhode Island, USA.

${ }^{2}$ Warren Alpert Medical School of Brown University, Brown University, Providence, Rhode Island, USA.

${ }^{3}$ Brown University and Department of Orthopaedic Surgery, Rhode Island Hospital, Providence, Rhode Island, USA.
} 
alization of infection via either [1] two or more positive cultures of phenotypically identical organisms, or [2] a visually discernible sinus tract communicates from the prosthesis to the skin and is not caused by aseptic failure. Pre-operative minor criteria include elevated serum $\mathrm{C}$-reactive protein (CRP) or erythrocyte sedimentation rate (ESR), elevation of synovial white blood cell (WBC) count or change on leukocyte esterase test strip, elevation of synovial polymorphonuclear leukocytes, and as of 2018, elevated alpha defensin. If these pre-operative minor criteria are inconclusive in diagnosing PJI, intra-operative criteria of positive histologic analysis of peri-prosthetic tissue, positive purulence, or a single positive culture result can be used to confirm or deny infection.

Historically, the MSIS criteria were introduced in 2011 as the result of a collaborative review of laboratory findings designed to streamline and standardize diagnosis of PJI [11]. These criteria were re-evaluated in 2018 testing the efficacy of established criteria as well as other possible inflammatory markers, resulting in the addition of alpha-defensin to the minor criteria list [12]. These new criteria established a point scoring system for minor criteria based on beta coefficients from regression analysis to determine infection. If the preoperative score is inconclusive, intra-operative criteria are then considered. Table 1 shows the major and minor criteria

Table 1. 2018 Modified Musculoskeletal Infection SocIETy SCORING Definition FOR PERI-PROSTHETIC JOINT INFECTION

\begin{tabular}{|c|c|c|c|}
\hline \multicolumn{3}{|c|}{ Major criteria (need only one) } & Conclusion \\
\hline \multicolumn{3}{|c|}{$\begin{array}{l}\text { Two positive cultures of the same organism } \\
\text { Sinus tract communicating to joint } \\
\text { or visualization of the prosthesis }\end{array}$} & Infected \\
\hline \multicolumn{2}{|c|}{ Minor criteria (pre-operative) } & Score & Conclusion \\
\hline Serum & $\begin{array}{l}\text { Elevated } \\
\quad \text { CRP }(>1 \mathrm{mg} / \mathrm{dL}) \text { or }\end{array}$ & 2 & $\geq 6$ infected \\
\hline \multirow[t]{2}{*}{6 infected } & $\begin{array}{l}\text { D-dimer } \\
\quad(>860 \mathrm{ng} / \mathrm{mL})\end{array}$ & & \\
\hline & $\begin{array}{l}\text { Elevated } \\
\quad \text { ESR }(30 \mathrm{~mm} / \mathrm{h})\end{array}$ & 1 & $\begin{array}{l}2-5 \text { possible } \\
\text { infected }\end{array}$ \\
\hline \multirow[t]{5}{*}{ Synovial } & $\begin{array}{l}\text { Elevated WBC count } \\
\quad(>3000 \text { cells/mcg/L }) \\
\text { or }\end{array}$ & 3 & \\
\hline & $\mathrm{LE}(++)$ & & $\leq 1$ not \\
\hline & $\begin{array}{l}\text { Positive alpha-defensin } \\
\quad(\text { signal-to-cutoff } \\
\text { ratio }>1)\end{array}$ & 3 & \\
\hline & $\begin{array}{l}\text { Elevated synovial PMN } \\
\text { percent }(>80 \%)\end{array}$ & 2 & \\
\hline & $\begin{array}{l}\text { Elevated synovial CRP } \\
\quad(>6.9 \mathrm{mg} / \mathrm{L})\end{array}$ & 1 & \\
\hline
\end{tabular}

\begin{tabular}{lcc}
\hline Intra-operative scoring criteria & Score & Conclusion \\
\hline Positive histology & 3 & $\geq 6$ infected \\
Positive purulence & 3 & $4-5$ inconclusive \\
Single positive culture & 2 & $\leq 3$ not infected \\
\hline
\end{tabular}

$\mathrm{CRP}=\mathrm{C}$-reactive protein; $\mathrm{ESR}=$ erythrocyte sedimentation rate; $\mathrm{WBC}=$ white blood cell; $\mathrm{PMN}=$ polymorphonuclear leukocyte. along with their associated point values and corresponding conclusion on infection.

The 2018 criteria reported a sensitivity of $97.7 \%$ and a specificity of $99.5 \%$, but notes that the criteria may struggle to identify infection in patients with an adverse local tissue reaction or an infection with low virulence organisms, issues that will be discussed further in this review [12].

The biggest shortcoming of the MSIS system is that its major diagnostic criteria rely on methodology that does not reflect the urgency of treatment necessitated for infection. For example, diagnosing PJI using the MSIS criteria relies on obtaining a culture of the joint to confirm the final diagnosis of infection, because this allows for visualization of the offending organism.

A culture is required even with other secondary tests, because it can determine micro-biologic resistance profiles and antibiotic susceptibility [13]. Certain slow growing bacteria, however, such as Cutibacterium acnes (C. acnes), can require up to two weeks of incubation to confirm infectious etiology [9]. This leads to delays in diagnosis, treatment, and increased complications. In addition, accurate bacterial identification can be obscured because of contamination by commensal bacteria and contamination between plates during storage and incubation [14,15].

To compensate for the time required to culture organisms, patients are treated prophylactically with broad spectrum antibiotic agents [16], a problematic practice with rising rates of antibiotic resistant strains of pathogens [17]. Specific pathogen identification and an antibiotic resistance profile are often unknown before deciding on intervention, which can lead to treatment with antibiotic agents typically reserved for more virulent strains [16].

With regard to MSIS minor criteria, current research demonstrates both promising and disappointing results. Methods using the detection of biomarkers indicative of an inflammatory response to an infection can be inconclusive at differentiating PJI from aseptic loosening [18,19]. Such biomarkers, measured in the serum and synovial fluid, include CRP detection, leukocyte counts and differential, and measurement of interleukin (IL)-6 levels. Other biomarkers such as D-dimer and fibrinogen indicate coagulation as a marker of possible infection.

While the MSIS is considered the gold standard of diagnosis for PJI, the aforementioned markers have low specificity for PJI. As such, confirming a positive result requires secondary testing, which slows the speed of results and can be subject to false negative and positive results that can be seen in culturing methods.

Last, although MSIS criteria do not differ between joints, total shoulder arthroplasty (TSA) has a higher probability of infection with low virulence pathogens such as $C$. acnes, suggesting that "joint-specific" clinical guidelines might be a consideration. [9,20,21]. Overall, problems with detecting PJI and pathogen identification based on the criteria listed above demonstrate the need for more time sensitive and accurate diagnostic methods.

\section{Culturing}

Current culturing methodology uses samples of explant, tissue, or joint aspiration fluid that are incubated on agar plates under aerobic and anaerobic conditions to identify 
offending pathogens. As one of the two major MSIS criteria, culturing allows direct visualization of bacteria. In this system, two positive cultures of phenotypically identical organisms provide evidence that a specific pathogen is present. The growth of bacteria in culture, however, does not necessarily mean an infection is present. Various commensal bacteria live in and on the human body [22], meaning a single positive culture may not definitively indicate a PJI. This method can have a sensitivity as low as 9.7\% [23]. Even two or more positive tissue cultures can have a sensitivity as low as $40.74 \%$ [24].

The low virulence of Cutibacterium spp. can cause infections with reduced clinical symptoms, normal laboratory panels, and reduced values of conventional tests for PJI [9]. Diagnosis in this setting is challenging and can delay treatment [9]. Synovial fluid cultures had only a $49 \%$ sensitivity for PJI. Renz et al. [9] noted that peri-implant tissue culture had a sensitivity of $74 \%$, while explant sonication improved sensitivity to $81 \%$. In addition, successfully culturing C. acnes requires incubation under anaerobic conditions for up to 14 days $[9,25]$, making it a difficult pathogen to identify correctly and manage.

For TSA, culturing almost always returns monomicrobial results, most often $C$. acnes $[9,22]$ although non-virulent microbiota are known also to be present [26]. Quantifying the interior bacterial load, differentiating between epidermal and pathogenic $C$. acnes during sampling, and the chance of sample contamination can pose potential problems for diagnosing a PJI after TSA.

Primarily, C. acnes colonizes pilosebaceous glands that are much more prevalent on the shoulder and back than the hip and knee. It is seen less frequently in total hip arthroplasty and total knee arthroplasty (THA and TKA). In THA and TKA, culture duration of 14 days shows no benefit compared with shorter culturing periods, except more frequent growth of contaminants [14]. Interestingly, true positive infections with $C$. acnes in this setting grew positive cultures sooner (mean of five days) rather than later (greater than 11 days). Of patients with $C$. acnes growth, $80 \%$ had only one positive culture and cultures were polymicrobial, possibly indicating contamination [14]. This reinforces previous findings [15] that extended culturing durations up to 14 days are more likely to show a false positive from non-virulent pathogens or possible contamination.

Kheir et al. [25] found that over a large cohort [ $n=711$ ] of hip and knee PJIs, the mean number of samples needed to yield a minimum of two positive cultures (as per MSIS requirement) was four samples. Pathogens not within the five most prevalent organisms (Staphylococcus aureus, coagulase negative Staphylococcus spp. [CoNS], Streptococcus spp., Enterococcus spp., and gram negative bacilli) required an average of seven samples, however. For C. acnes, the mean was 10, and for Escherichia. coli, the mean was 25. This outlier result could be because of the propensity of E. coli to form a protective biofilm, which hinders its acquisition for culture.

In these cases, sonication fluid culture, an alternative to conventional methods, which removes adherent bacteria from explants, shows improved results compared with classical culturing, which may miss bacteria in biofilms [25]. Sensitivity and specificity of sonication fluid culture were $77.05 \%$ and $98.11 \%$ compared with $55.74 \%$ and $94.34 \%$ for traditional peri-prosthetic tissue culture [27]. Yan et al. [28] examined sonication fluid cultures and found similar results, with sensitivity of $73.1 \%$ and specificity of $96.0 \%$. These results, however, are not consistent with another study by Dudareva et al. [29] showing a reduction in sensitivity from $97 \%$ to $76 \%$ and specificity from $69 \%$ to $57 \%$ with sonication compared wit tissue culture.

Inagaki et al. [30] reported similarly high statistics for conventional culturing including one positive culture, producing $88.3 \%$ sensitivity, while two positive cultures decreased the sensitivity to $80 \%$. Decreased sensitivity with two cultures could point to the presence of contamination and the inconsistency of relying on culturing to confirm PJI. Culturing of sonication fluid should be explored further to determine its utility in the diagnosis of biofilm-forming bacteria versus conventional methods.

Other novel methods, such as tissue placement in blood culture bottles, can be financially more efficient, isolate additional micro-organisms [31], and show sensitivity and specificity similar to sonication [28]. Blood culture bottles could help visualize more non-virulent organisms that can cause less evident PJI [31]. Although time consuming, when conventional culture was combined with polymerse chain reaction (PCR) and vial culture, the specificity reached $98.57 \%$, making it a valuable tool for ruling out infection [24].

Similar to other methods of detection, culturing can accurately detect infections with virulent pathogens such as S. aureus with few plates within a short period. Less virulent infections with species such as CoNS and $C$. acnes require more efficient methods to diagnose PJI accurately. Less virulent pathogens and those using protective biofilms necessitate more samples to produce positive results, although increased number of cultures can decrease sensitivity. Further, culturing is susceptible to contamination, because positive cultures in an aseptic patient are often contaminated with CoNS [23]. Culturing is time consuming, and results and protocols can vary between pathogens and sample sites, but remains the only method of PJI detection outside of the presence of a sinus tract that allows direct visualization of infection.

\section{PCR diagnosis}

The PCR] is used to detect pathogen deoxyribonucleic acid (DNA) in synovial fluid by amplifying sequences of the bacterial genome. By applying this technique to multiple DNA sequences, a multiplex PCR (mPCR) can look for the presence of many pathogens and can be used as an alternative to traditional culturing to identify bacterial presence down to the specific genus and species for bacterial sequences already in a database [32]. The PCR is primarily compared with culturing because of the similar utility in isolating species and identifying antibiotic resistance. Results of PCR, however, are available much faster (within $6 \mathrm{~h}$ in some protocols [32]), and require a smaller amount of synovial fluid [33].

Shotgun metagenomic sequencing, a new form of PCR where every nucleic acid is sequenced and compared with a database to identify genetic traits of organisms, showed improved sensitivity compared with synovial fluid culture: 68.5\% compared with 54.0\%. Metagenomics found bacteria in $94.5 \%$ of culture-positive fluid samples, and most of the identified microorganisms were partially or exactly 
concordant with culture results. Sequencing also detected bacteria in $40.0 \%$ of culture-negative sonicate fluid, however. More than half of these results came from patients who had received pre-operative antibiotic agents. Sequencing can detect bacteria even when a patient has already received antibiotics, which can adversely affect culturing results [34].

The mPCR also showed no adverse effects to results of patients who had received pre-operative antibiotic agents [32]. Sensitivity for mPCR was $71.1 \%$, while culturing was $84.2 \%$, but when these procedures are combined, sensitivity reached $92.1 \%$ [32]. Because sequencing targets specific nucleic acids, metagenomic analysis is limited by the amount of host DNA present in the sample. This can be problematic when thresholds are lowered to increase detection, ultimately increasing the specificity but lowering the sensitivity because of DNA contamination [35].

The mPCR showed $85.6 \%$ agreement on bacterial presence with culturing. It also detected more Cutibacterium spp. and $C o N S$, indicating its utility in detecting low virulence organisms. Low virulence infections currently missed using the standard MSIS criteria could have diagnostic improvements by including a PCR procedure as part of PJI diagnosis [32]. Fastidious and difficult to culture micro-organisms such as $C$. pneumonia, S. maltophilia, and B. melitensis were also detected by mPCR [36]. Moshirabadi et al. [36] showed vast improvement in sensitivity (from $31.6 \%$ up to $97.4 \%$ ), compared with culturing methods.

Unlike mPCR, broad-range PCR as well as real-time ribonucleic acid (RNA) PCR utilize primers for the 16S rRNA sequence, which is highly conserved among bacterial species and not present in the human genome [37]. Broad-range PCR primers target the genomic sequence less specifically, giving a wider range of amplified sequences than real-time PCR, which has specific primers that amplify more limited sequences [38]. Fang et al. [37] compared the utilization of DNA qPCR with RNA qPCR to determine which proved a better diagnostic tool for detecting PJI. The RNA-based qPCR was found to be less sensitive $(73.6 \%)$ than its DNA counterpart $(81.5 \%)$ This is more specific than DNA and more sensitive than culture. Even though DNA qPCR provided the highest sensitivity, this ultimately creates problems with DNA contamination. These findings indicated that RNA based qPCR is a better diagnostic test, having the highest accuracy at $85.9 \%$.

Real-time RNA PCR results agreed with culturing for joint fluid, peri-prosthetic tissue, and sonicated fluid $77.6 \%$, $61.2 \%$, and $83.6 \%$ of the time, respectively. The sensitivities for joint fluid $(83.0 \%)$ and sonicated fluid (84.9\%) were both higher than the sensitivities of their culturing counterparts. The specificities of these samples, however, followed the opposite trend. Peri-prosthetic tissue had a much lower sensitivity $(34.0 \%)$, indicating little promise in its diagnostic abilities [39].

Peri-prosthetic joint infection is not only caused by bacterial colonization but can also occur from fungal presence. Real-time PCR utilizing additional primers such as $28 \mathrm{~S}$ rRNA [40] or 18S rRNA [41] allow for the detection of fungal and eukaryotic presence. The PCR run with an $18 \mathrm{~S}$ rRNA primer on a synovial fluid sample taken pre-operatively had a sensitivity of $55.6 \%$ and specificity of $82.0 \%$ in detection of either bacterial or fungal PJI. These values were compara- tively less than or equal to those found for culture and CRP values of the same samples. Even with the additional fungal primer, pre-operative synovial fluid testing did not add diagnostic value [41].

Kuo et al. [40], however, found both high sensitivity (100\%) and specificity (99.5\%) for PCR run on synovial fluid samples collected intra-operatively. These values were significantly higher than the biomarkers used for comparison. In addition, using RNA primers over DNA primers reduced the false positive rate to $4 \%$ [40]. Reaching such high sensitivity and specificity helps to combat one of the major problems with PCR (false positive results because of contamination), thus demanding further investigation into this combination of primers.

PCR shows promise as an alternative to culturing for direct identification of bacterial species and antibiotic resistance. Different PCR methodologies have been shown to be unaffected by recent administration of antibiotic agents, unlike culturing, and allow for identification of difficult to culture pathogens. The PCR, however, can also detect small bacterial loads of "silent" colonizations of prostheses without clinical relevance, reinforcing the idea that bacteria presence does not always lead to infection [33].

The PCR can also be misleading in polymicrobial infections because sequence analysis is unable to distinguish properly the nucleic acid peaks because of intertwining from multiple species $[39,40]$. Missing other pathogenic bacteria in an infection can cause mistreatment or misadministration of antibiotic agents. Ultimately, PCR is a costly diagnostic test, each sample costing several hundred US dollars [35], which creates further problems if the genetic material in the sample is not read easily.

\section{Biomarkers}

Biomarkers help in the diagnosis of PJI, as they are signals of the body's response to infection. The most commonly used biomarkers, CRP and erythrocyte sedimentation rate (ESR), indicate an inflammatory response by the immune system in relation to the presence of foreign pathogens. Coagulation biomarkers, such as plasma fibrinogen and D-dimer, are being investigated currently as possible indicators of infection. These markers help determine presence of a blood clotting response in the surgical area caused by infection. Not only can markers of human immune responses be used to determine infection, but also studies indicate that identification of bacterial biomarkers confirms their presence [42]. These markers include cell wall components like lipoteichoic acid present in gram-positive cell walls and biologic byproducts of bacterial metabolism.

The CRP is an inflammatory biomarker used in distinguishing PJI from aseptic loosening. Studies have shown that CRP cannot stand alone as a diagnostic tool for distinguishing PJI, but it is helpful when paired with other inflammatory markers. Schiffner et al. [19] found that at the $1 \mathrm{mg} / \mathrm{dL}$ threshold for CRP, there was a $65 \%$ sensitivity and a $56 \%$ specificity. When CRP is measured from synovial fluid, sensitivity and specificity both increase to $88 \%$ and $82 \%$, respectively, suggesting that synovial CRP may be a better diagnostic test for PJI than serum CRP. Alone, however, regardless of source, CRP is not specific enough to distinguish PJI from aseptic loosening $[19,43]$. Despite this, CRP is 
a useful complementary test, because patients with higher CRP levels had significant correlation to having more positive intra-operative cultures [44].

Along with CRP, ILs are used to help determine PJI and are often paired with CRP testing. Alone, IL-6 level measurements were shown to have an $86.7 \%$ sensitivity and $89.5 \%$ specificity for PJI, but when paired with serum CRP testing, sensitivity fell to $73.7 \%$ while specificity increased to $100 \%$ [45]. This decrease in sensitivity while an increase in specificity is a common problem in pairing biomarker tests [45], because a decline in sensitivity correlates with an increase in false-negative test results, missing cases of PJI. Along with CRP, ILs are used to help determine PJI and are often paired with CRP testing. This is the reason other ILs such as IL-16, IL-18, and cysteine rich with EGF-like domains 2 (CRELD2) are being investigated as better indicators. Chen et al. [46] found that IL-16 and IL-18 and CRELD2 had statistical significance as overall better indicators in PJI detection than CRP and leukocyte count.

Other inflammatory biomarkers, including presepsin, a soluble fraction of CD14, have been shown to have positive linear correlation with both CRP and IL-6, making them comparable and possible second-line tests [47]. SaNgasoongsong et al. [48[ determined that procalcitonin (PCT) had a $65 \%$ sensitivity and $91.7 \%$ specificity for PJI when levels were determined from serum, but the sensitivity increased to $80 \%$ when PCT was measured from the synovial fluid.

Along with biomarkers for inflammatory response, indicators of coagulation such as D-dimer and fibrinogen levels can be used to detect PJI. Fibrinogen has been found to be a more promising marker with sensitivity and specificity similar to that of traditional inflammatory markers. Plasma fibrinogen sensitivity and specificity range from $76.3-81.0 \%$ and 25.0-86.2\%, respectively [49,50]. Plasma fibrinogen has even been shown to have sensitivity as high as $90 \%$ when the threshold was lowered to $519 \mathrm{mg} / \mathrm{dL}$, but the specificity of the test still suffered, reaching only $34 \%$ [50]. In addition, plasma fibrinogen has a negative predictive value of $98.3 \%$, making it a reasonable marker for ruling out PJI [51].

In comparison, D-dimer had much lower values of $64.5 \%$ sensitivity and $65.0 \%$ specificity [49]. D-dimer, when combined with other biomarker tests, is seen to have an increase in specificity, because D-dimer on its own had sensitivity and specificity values of $67.44 \%$ and $44.09 \%$. When paired with fibrinogen and CRP tests, however, the specificity increased to $90.14 \%$ and sensitivity dropped to only $56.10 \%$ [52]. Overall, plasma fibrinogen appears to be equally effective as inflammatory markers in ruling out PJI, but the clinical relevancy of D-dimer as an informatory biomarker seems to be less promising.

Not only can biomarkers of host immune response be used to determine infection, but biologic markers of the bacteria also can provide an even clearer picture. Host biologic markers quantify inflammatory or coagulation responses, which are host responses to an infection, but the presence of these markers becomes clouded when a patient has inflammatory conditions like arthritis, or if the inflammation is simply because of irritation from aseptic failure [53].

On the other hand, bacterial biomarkers include the metabolic product D-lactate, which is produced almost exclusively by bacteria [54] and whose synovial fluid concen- tration can be measured spectrophotometrically. Yermak et al. [42] found that using these D-lactate concentrations yielded $86.4 \%$ sensitivity and $81.7 \%$ specificity, values similar to those for synovial leukocyte count.

Bacteria can also be visualized using these markers, as done in fluorescence in-situ hybridization (FISH), which stains parts of the peri-prosthetic membranes that have bacterial presence. Visualizing bacteria via FISH has a $95 \%$ sensitivity and $85.42 \%$ specificity related to clinical findings, and higher values of $95.12 \%$ and $87.23 \%$ when correlated with histopathologic findings [55].

Monoclonal mouse antibodies are also used to visualize bacteria, because radio-labeled mouse antibody targets lipoteichoic acid (LTA) in gram-positive cell walls [18]. On imaging the infected mice, a weak positive correlation between the bioluminescent signal and standardized uptake values was seen with an $R$ value of $0.767\left(R^{2}=0.588\right)$, indicating specificity of the radio-labeled antibody, but little direct association between the probes [18]. These bacterial biomarkers give a true determination of the bacterial presence in a surgical site, unlike inflammatory and coagulation markers that detect the presence of a response.

\section{Alpha defensin}

Synovial fluid levels of alpha defensin have emerged as an adjunct biomarker indicative of local reaction to infection. Alpha defensin is a peptide released by neutrophils with a broad range of anti-pathogenic coverage against bacteria, fungi, and enveloped viruses. Alpha defensins induce pore formation in microbial membranes leading to depolarization and eventual inactivation [56]. As proven with its addition to the 2018 update of the MSIS criteria, clinical data suggest measuring alpha defensin levels can be effective in the diagnosis of PJI.

There are two types of tests available for synovial levels of alpha defensin: The synovasure alpha defensin lateral flow (ADLF) test, and an enzyme-linked immunosorbent assay (ELISA). The results of the ADLF test are available after 10 min while the ELISA results are available the next day after laboratory analysis.

The role of apha defensin in the local immune response to an infection makes it a prime candidate in the diagnosis of PJI. With a higher specificity than sensitivity in almost every study examined, measurement of alpha defensin level is useful as a confirmatory test, but not a good procedure to rule out diagnosis of PJI or as the sole detection procedure $[5,8]$. When combined with culturing, sensitivity and specificity reach $96 \%$ and $100 \%$, respectively [57]. Alpha defensin may miss infections with low-virulence organisms such as coagulase negative CoNS or C. acnes, with up to an $80 \%$ false negative rate $[21,58]$.

Synovial levels of alpha defensin are unaffected by recent antibiotic therapy in patients [56,58], giving it an advantage over biomarkers such as CRP [43]. Synovasure ADLF showed statistical improvement over MSIS minor criteria including ESR/CRP level, pre-operative culture (1 positive), and synovial cell count $[23,60]$.

Following staged treatment of PJI with insertion of an antibiotic spacer, alpha defensin testing showed low sensitivity but high specificity, furthering the conclusion that the test can be used effectively to confirm diagnosis $[61,62]$. 
In patients with adverse local tissue reaction secondary to physical prosthetic failure, Synovasure ADLF had a sensitivity and specificity of $100 \%$ and $68 \%$, suggesting that alpha defensin may be prone to false positives in this setting [63]. Therefore, when metallosis occurs, alpha defensin testing may be unable to distinguish between septic and aseptic failure.

In the current review, six studies on the Synovasure ADLF test are grouped together because of their similarity in criteria, time period, and samples, while three on ELISA are deemed comparable. The average sensitivity and specificity of the ADLF test were $81.45 \%$ and $94.53 \%$, respectively. The average sensitivity and specificity of the ELISA were $84.7 \%$ and $91.7 \%$ (Table 2).

In summary, alpha defensin testing remains a useful tool for the diagnosis of PJI, but its strengths and weaknesses should be noted. The averages of several studies of Synovasure ADLF resulted in a specificity of $95 \%$, making this rapid and easy test useful to confirm the presence of infection. Alpha defensin levels have been shown to be unaffected by recent antibiotic therapy and can be used in synovial fluid contaminated with blood [8]. The lower sensitivity and possible false negatives because of low virulence pathogens, however, show that this test is not optimal for detecting infections and should not be used independently of other tests.

The current cutoff for Synovasure may prevent its use in differentiating PJI from gout, pseudogout, and other inflammatory arthropathies [4]. Further, alpha defensin testing does not provide any information about what pathogen is present, leading to broad coverage antibiotic use before return of culture results, which can narrow down the proper antibiotic therapy [15].

\section{Leukocytes}

As effector cells of the innate and adaptive immune systems, leukocytes are a critical and functional response to infection. Levels of leukocytes in the blood or synovial fluid, therefore, can be elevated both acutely and chronically to combat a pathogen. Leukocyte levels reflect the presence of inflammation as a result of infection. Several tests have been designed to detect leukocyte levels including the leukocyte esterase (LE) test, histopathologic tissue analysis, and radiolabeled scans.

The LE test rapidly detects the level of enzymes released by neutrophils in synovial fluid. Studies examining LE test strips found sensitivities ranging from $74.22 \%$ to $80.5 \%$ and specificities above $92.7 \%[20,64]$. The best sensitivity results were from a Synovasure lateral flow immunoassay with sensitivity of $91.95 \%$ [64]. Like other tests, in the context of TSA, LE statistics were poorer with a sensitivity of $50 \%$ and a specificity of $87 \%$. Like alpha defensin testing, LE test strips have low sensitivity and high specificity, making LE a poor method for PJI screening, but a better confirmatory test.

Further, the test only detected pathogens in three of 15 patients specifically infected with $C$. acnes, and also had several invalid results because of contamination with blood or insufficient synovial fluid levels [20]. These problems with the LE test strip utilization make it difficult to use as a primary diagnostic test for PJI.

Intra-operatively, the LE test had a sensitivity and specificity of $100 \%$ and $93.8 \%$, respectively, compared with $85.7 \%$ and $94.9 \%$ for frozen section histology in THA [65]. The time from sampling to the results being returned to the surgeon was on average $20.1 \mathrm{~min}$ for LE and $27.2 \mathrm{~min}$ for frozen section, a useful increase in efficiency, compared with some of the previous methods, during an operation [65].

The LE test strips can be contaminated by blood, leading to invalid readings even when high amounts of WBCs and LE are present in synovial fluid. False positive results can also occur in the setting of physical failure where metal-wear debris interferes with the test or an adverse local tissue reaction increases the level of leukocytes present [65]. Although the test is rapid, inexpensive, and commercially available, its high invalidation rate, up to $11.9 \%$, and low sensitivity make it a poor test to rule out infection [64].

Histopathologic tissue analysis detects leukocytic infiltration as a marker of infection. One study, however, showed no statistical relationship between intra-operative histopathologic examination of neutrophils, which diagnosed $8 \%$ of infections, and post-operative hematoxylin and eosin analysis, which diagnosed $28 \%$ [66], calling into question the validity of this methodology.

Another method of measuring leukocytes is synovial fluid cell counts. Comparing effectiveness between studies is difficult because each uses a different cutoff value to determine the presence of infection. Synovial leukocyte cell counts produce promising results, but the lack of standardization limits the diagnostic relevance.

Although WBC ranges are more standardized in the settings of THA and TKA, they cannot be extrapolated to

Table 2. Summary of Reported Alpha Defensin Test Results

\begin{tabular}{|c|c|c|c|c|c|c|}
\hline Author & Sample size & Sensitivity & Specificity & $N P V$ & $P P V$ & Type of test \\
\hline Kanwar et al. & 35 & 97.1 & 97.1 & 97.1 & 97.1 & ELISA \\
\hline Renz et al. & 212 & 84.4 & 96.4 & 95.8 & 86.4 & ADLF \\
\hline de Saint Vincent et al. & 42 & 88.9 & 90.6 & 96.7 & 72.7 & ADLF \\
\hline Balato et al. & 51 & 87.5 & 97.1 & 94.4 & 93.3 & ADLF \\
\hline Riccio et al. & 40 & 85.0 & 96.9 & & & ADLF \\
\hline Sigmund et al. & 101 & 69.0 & 94.0 & 88.3 & 83.3 & ADLF \\
\hline Ding et al. & 70 & 73.9 & 92.2 & 77.8 & 90.4 & ADLF \\
\hline Kelly et al. & 39 & 82.0 & 82.0 & 92.0 & 64.0 & ELISA \\
\hline Ecker et al. & 105 & 75.0 & 96.0 & 93.0 & 86.0 & ELISA \\
\hline Average (Synovasure) & & 81.5 & 94.5 & 90.6 & 85.2 & \\
\hline Average (ELISA) & & 84.7 & 91.7 & 94.0 & 82.4 & \\
\hline
\end{tabular}

$\mathrm{NPV}=$ negative predictive value; $\mathrm{PPV}=$ positive predictive value; ADLF $=$ alpha defensin lateral flow test; ELISA=enzyme-linked immunosorbant assay 
TSA [67]. Further, although one might assume that low grade shoulder infections with pathogens such as $C$. acnes would produce fewer cell counts, they are, in fact, associated with high leukocyte levels [67]. Inagaki et al. [30] found that almost every case of septic knee failure had 5+ neutrophils per 400x magnification in a high power field, indicating its utility visualizing both virulent and non-virulent infections. The $5+$ neutrophils under histologic analysis of TKA produced $96.7 \%$ sensitivity and $100 \%$ specificity in detection of either bacterial or fungal PJI. Neutrophil count does not provide information regarding the degree of infection or bacterial load.

Finally, radio-labeled assays can detect leukocyte accumulation. Blanc et al. used labeled leukocyte scintigraphy [LS] and found $72 \%$ sensitivity and $60 \%$ specificity [68]. The LS results were unaffected by current antibiotic treatment or type of pathogen, but the process is expensive and time consuming. Falstie-Jensen et al. [69] found a sensitivity of $18 \%$ and a specificity of $100 \%$ in TSA using WBC/bone marrow single-photon emission computed tomography. The C. acnes infections, however, were not detectable by this method.

Leukocyte levels are increased in response to infection. Although secondary to detecting pathogen presence, measuring leukocyte levels are useful in the diagnosis of PJI. Similar to other secondary methods, however, leukocyte levels are not specific for PJI and can be increased because of other causes of inflammation such as gout [70].

\section{Conclusion}

The methodologies used currently in the diagnosis and detection of PJI have both benefits and drawbacks. Biomarkers can give inaccurate results when the patient has low virulence infections or complications such as metallosis or inflammatory diseases, which can raise biomarker levels without the presence of infection. Culturing becomes problematic because of the difficulty in getting a representative swab as well as the often long necessary culture time and the low sensitivity for one positive culture in diagnosis. Other tests, such as PCR and radio-labeled WBC can be expensive to perform. For more affordable tests, such as LE strips, the results are easily invalidated by blood contamination and low sensitivity.

The current clinical "gold standard," the MSIS criteria, does not differentiate between surgical sites, and it relies mainly on culturing as a diagnostic tool. Future directions include direct visualization of bacteria at a surgical site, allowing for real quantification of bacterial presence instead of relying on antibodies and fluorescent markers. This form of rapid visualization assay could provide a solution to many of the problems other tests have with detecting PJI, including low-virulence infections, ambiguous results because of metallosis and inflammatory diseases, and getting an accurate representation of the site in question. Overall, continued research into all methods of PJI detection as well as the determination criteria is vital to most efficiently diagnose and manage PJI.

\section{Acknowledgments}

We would like to thank Diane N. Weiss and the Sipprelle Family Foundation for their continued support.

\section{Author Disclosure Statement}

Dr. Garcia holds equity in BI Medical, LLC; Dr. Born, holds equity in BI Medical, LLC. and is a stock holder in BioIntraface, Inc, and Illuminoss, LLC. For the remaining authors, no competing financial interests exist.

\section{References}

1. Sebastian S, Malhotra R, Dhawan B. Prosthetic joint infection: A major threat to successful total joint arthroplasty. Indian J Med Microbiol 2018;36:475-487.

2. Guan H, Fu J, Li X, et al. The 2018 new definition of periprosthetic joint infection improves the diagnostic efficiency in the Chinese population. J Orthop Surg Res 2019; $14: 151$.

3. Kurtz SM, Lau E, Watson H, et al. Economic burden of periprosthetic joint infection in the United States. J Arthroplasty 2012; 27(Suppl 8):61-65.e1.

4. Adams JR, Schwartz AJ. False-negative synovial alphadefensin. Arthroplast Today 2017;3:239-241.

5. Sigmund IK, Holinka J, Lang S, et al. A comparative study of intraoperative frozen section and alpha defensin lateral flow test in the diagnosis of periprosthetic joint infection. Acta

6. Bingham JS, Salib CG, McQuivey K, et al. An evidencebased clinical prediction algorithm Orthop 2019; 90: 105-110. for the Musculoskeletal Infection Society minor criteria. J Arthroplasty 2018 33:2993-2996.

7. Kwiecien G, George J, Klika AK, et al. Intraoperative frozen section histology: Matched for Musculoskeletal Infection Society criteria. J Arthroplasty 2017;32:223-227.

8. Renz N, Yermak K, Perka C, Trampuz A. Alpha defensin lateral flow test for diagnosis of periprosthetic joint infection: Not a screening but a confirmatory test. J Bone Joint Surg Am 2018;100:742-750.

9. Renz N, Mudrovcic S, Perka C, Trampuz A. Orthopedic implant-associated infections caused by Cutibacterium spp.-A remaining diagnostic challenge. PLoS One 2018; 13:e0202639.

10. Parvizi J, Gehrke T. Definition of periprosthetic joint infection. J Arthroplasty 2014;29:1331.

11. Parvizi J, Zmistowski B, Berbari EF, et al. New definition for periprosthetic joint infection: From the Workgroup of the Musculoskeletal Infection Society. Clin Orthop Relat Res 2011;469:2992-2994.

12. Parvizi J, Tan TL, Goswami K, et al. The 2018 Definition of periprosthetic hip and knee infection: An evidencebased and validated criteria. J Arthroplasty 2018;33:13091314.e2.

13. Romanò CL, Khawashki HA, Benzakour $\mathrm{T}$, et al. The W.A.I.O.T. definition of high-grade and low-grade periprosthetic joint infection. J Clin Med 2019;8.

14. Klement MR, Cunningham DJ, Wooster BM, et al. Comparing standard versus extended culture duration in acute hip and knee periprosthetic joint infection. J Am Acad Orthop Surg 2019;27:e437-e4443.

15. Butler-Wu SM, Burns EM, Pottinger PS, et al. Optimization of periprosthetic culture for diagnosis of Propionibacterium acnes prosthetic joint infection. J Clin Microbiol 2011;49:2490-2495.

16. Sousa R, Abreu MA. Treatment of prosthetic joint infection with debridement, antibiotics and irrigation with implant retention-a narrative review. J Bone Jt Infect 2018; 3: $108-117$. 
17. Ravi S, Zhu M, Luey C, Young SW. Antibiotic resistance in early periprosthetic joint infection. ANZ J Surg 2016;86: 1014-1018.

18. Pickett JE, Thompson JM, Sadowska A, et al. Molecularly specific detection of bacterial lipoteichoic acid for diagnosis of prosthetic joint infection of the bone. Bone Res 2018;6:13.

19. Schiffner E, Latz D, Thelen S, et al. Normal CRP and WBC values in total hip arthroplasty [THA] with signs of loosening. Do we need a joint aspiration? J Clin Orthop Trauma 2019;10:566-570.

20. Zahar A, Lausmann C, Cavalheiro C, et al. How reliable is the cell count analysis in the diagnosis of prosthetic joint infection? J Arthroplasty 2018;33:3257-3262.

21. Unter Ecker N, Koniker A, Gehrke T, et al. What is the diagnostic accuracy of alpha-defensin and leukocyte esterase test in periprosthetic shoulder infection? Clin Orthop Relat Res 2019; 477:1712-1718.

22. Garcia DR, Deckey D, Haglin JM, et al. Commonly encountered skin biome-derived pathogens after orthopedic surgery. Surg Infect 2019; 20:341-350.

23. Balato G, Franceschini V, Ascione T, et al. High performance of $\alpha$-defensin lateral flow assay [Synovasure] in the diagnosis of chronic knee prosthetic infections. Knee Surg Sports Traumatol Arthrosc 201;26:1717-1722.

24. Stylianakis A, Schinas G, Thomaidis PC, et al. Combination of conventional culture, vial culture, and broad-range PCR of sonication fluid for the diagnosis of prosthetic joint infection. Diagn Microbiol Infect Dis [Internet]. 2018;92: 13-18.

25. Kheir MM, Tan TL, Ackerman CT, et al. Culturing periprosthetic joint infection: Number of samples, growth duration, and organisms. J Arthroplasty 2018;33:3531-3536.

26. Namdari S, Nicholson T, Abboud J, et al. Comparative study of cultures and next-generation sequencing in the diagnosis of shoulder prosthetic joint infections. J Shoulder Elbow Surg 2019;28:1-8.

27. Tani S, Lepetsos P, Stylianakis A, et al. Superiority of the sonication method against conventional periprosthetic tissue cultures for diagnosis of prosthetic joint infections. Eur J Orthop Surg Traumatol 2018;28:51-57.

28. Yan Q, Karau MJ, Greenwood-Quaintance KE, et al. Comparison of the diagnostic accuracy of periprosthetic tissue culture in blood culture bottles to that of prosthesis sonication fluid culture for diagnosis of prosthetic joint infection (PJI) by use of Bayesian latent class modeling and IDSA PJI criteria for classification. J Clin Microbiol 2018;56.

29. Dudareva M, Barrett L, Figtree M, et al. Sonication versus tissue sampling for diagnosis of prosthetic joint and other orthopedic device-related infections. J Clin Microbiol 2018;56. Available from: http://www.ncbi.nlm.nih.gov/ pubmed/30209185

30. Inagaki $\mathrm{Y}$, Uchihara $\mathrm{Y}$, Munemoto $\mathrm{M}$, et al. Correlation of histological and microbiological findings in septic and aseptic knee implant failure. Arch Orthop Trauma Surg 2019; 139:717-722.

31. van den Bijllaardt $W$, van der Jagt OP, Peijs $M$, et al. Culturing periprosthetic tissue in blood culture bottles results in isolation of additional microorganisms. Eur J Clin Microbiol Infect Dis 2019;38:245-252.

32. Sigmund IK, Windhager R, Sevelda F, et al. Multiplex PCR Unyvero i60 ITI application improves detection of lowvirulent microorganisms in periprosthetic joint infections. Int Orthop 2018;43:1891-1898.
33. Morgenstern C, Cabric S, Perka C, et al. Synovial fluid multiplex PCR is superior to culture for detection of lowvirulent pathogens causing periprosthetic joint infection. Diagn Microbiol Infect Dis 2018;90:115-119.

34. Yan Q, Wi YM, Thoendel MJ, et al. Evaluation of the CosmosiD bioinformatics platform for prosthetic jointassociated sonicate fluid shotgun metagenomic data analysis. J Clin Microbiol 2019;57.

35. Ivy MI, Thoendel MJ, Jeraldo PR, et al. Direct detection and identification of prosthetic joint infection pathogens in synovial fluid by metagenomic shotgun sequencing. J Clin Microbiol 2018;56.

36. Moshirabadi A, Razi M, Arasteh P, et al. Polymerase chain reaction assay using the restriction fragment length polymorphism technique in the detection of prosthetic joint infections: A multi-centered study. J Arthroplasty 2019;34: 359-364.

37. Fang XY, Li WB, Zhang CF, et al. Detecting the presence of bacterial DNA and RNA by polymerase chain reaction to diagnose suspected periprosthetic joint infection after antibiotic therapy. Orthop Surg 2018;10:40-46.

38. Patel A, Harris KA, Fitzgerald F. What is broad-range 16S rDNA PCR? Arch Dis Child Educ Pract Ed 2017;102: 261-264.

39. Huang Z, Wu Q, Fang X, et al. Comparison of culture and broad-range polymerase chain reaction methods for diagnosing periprosthetic joint infection: Analysis of joint fluid, periprosthetic tissue, and sonicated fluid. Int Orthop 2018; 42:2035-2040.

40. Kuo FC, Lu YD, Wu CT, et al. Comparison of molecular diagnosis with serum markers and synovial fluid analysis in patients with prosthetic joint infection. Bone Joint J 2018; 100-B:1345-1351.

41. Fink B, Steurer M, Hofäcker S, et al. Preoperative PCR analysis of synovial fluid has limited value for the diagnosis of periprosthetic joint infections of total knee arthroplasties. Arch Orthop Trauma Surg [Internet]. 2018; 138:871-878.

42. Yermak K, Karbysheva S, Perka C, et al. Performance of synovial fluid D-lactate for the diagnosis of periprosthetic joint infection: A prospective observational study. J Infect 2019; 79:123-129.

43. Plate A, Anagnostopoulos A, Glanzmann J, et al. Synovial C-reactive protein features high negative predictive value but is not useful as a single diagnostic parameter in suspected periprosthetic joint infection [PJI]. J Infect 2019;78: 439-444.

44. Akgün D, Müller M, Perka C, Winkler T. The serum level of $\mathrm{C}$-reactive protein alone cannot be used for the diagnosis of prosthetic joint infections, especially in those caused by organisms of low virulence. Bone Joint J 2018;100-B: 1482-1486.

45. Gallo J, Svoboda M, Zapletalova J, et al. Serum IL-6 in combination with synovial IL-6/CRP shows excellent diagnostic power to detect hip and knee prosthetic joint infection. PLoS One 2018;13:e0199226.

46. Chen MF, Chang CH, Yang LY, et al. Synovial fluid interleukin-16, interleukin-18, and CRELD2 as novel biomarkers of prosthetic joint infections. Bone Joint Res 2019; 8:179-188.

47. Marazzi MG, Randelli F, Brioschi M, et al. Presepsin: A potential biomarker of PJI? A comparative analysis with known and new infection biomarkers. Int J Immunopathol Pharmacol 2018;31:394632017749356. 
48. Sa-Ngasoongsong $\mathrm{P}$, Wongsak $\mathrm{S}$, Jarungvittayakon $\mathrm{C}$, et al. Comparison of synovial fluid and serum procalcitonin for diagnosis of periprosthetic joint infection: A pilot study in 32 patients. Biomed Res Int 2018;8351308.

49. Li R, Shao HY, Hao LB, et al. Plasma fibrinogen exhibits better performance than plasma D-dimer in the diagnosis of periprosthetic joint infection: A multicenter retrospective study. J Bone Joint Surg Am. 2019; 101 ... 613-619.

50. Klim SM, Amerstorfer F, Gruber G, et al. Fibrinogen-a practical and cost efficient biomarker for detecting periprosthetic joint infection. Sci Rep 2018;8:8802.

51. Xu C, Qu PF, Chai W, et al. Plasma fibrinogen may predict persistent infection before reimplantation in twostage exchange arthroplasty for periprosthetic hip infection. J Orthop Surg Res 2019;14:133.

52. Xu H, Xie J, Huang Q, et al. Plasma fibrin degradation product and D-dimer are of limited value for diagnosing periprosthetic joint infection. J Arthroplasty 2019;34:24542460.

53. Osei-Bimpong A, Meek JH, Lewis SM. ESR or CRP? A comparison of their clinical utility. Hematology 2007;12: 353-357.

54. Mazumdar S, Clomburg JM, Gonzalez R. Escherichia coli strains engineered for homofermentative production of D-lactic acid from glycerol. Appl Environ Microbiol. 2010; 76:4327-4336.

55. Lippmann T, Braubach P, Ettinger M, et al. Fluorescence in situ hybridization (FISH) for the diagnosis of periprosthetic joint infection in formalin-fixed paraffin-embedded surgical tissues. J Bone Joint Surg Am 2019;101:e5.

56. Lehrer RI, Lu W. $\alpha$-Defensins in human innate immunity. Immunol Rev 2012;245:84-112.

57. Kanwar S, Al-Mansoori AA, Chand MR, et al. What is the optimal criteria to use for detecting periprosthetic joint infections before total joint arthroplasty? J Arthroplasty 2018;33:S201-S204.

58. Scholten R, Visser J, Van Susante JLC, Van Loon CJM. Low sensitivity of «-defensin (Synovasure) test for intraoperative exclusion of prosthetic joint infection. Acta Orthop 2018;89:357-359.

59. Kelly MP, Darrith B, Hannon CP, et al. Synovial fluid alpha-defensin is an adjunctive tool in the equivocal diagnosis of periprosthetic joint infection. J Arthroplasty 2018; 33:3537-3540.

60. Riccio G, Cavagnaro L, Akkouche W, et al. Qualitative alpha-defensin versus the main available tests for the diagnosis of periprosthetic joint infection: Best predictor test? J Bone Jt Infect 2018;3:156-164.

61. Samuel LT, Sultan AA, Kheir M, et al. Positive alphadefensin at reimplantation of a two-stage revision arthroplasty is not associated with infection at 1 year. Clin Orthop Relat Res 2019;477:1615-1621.

62. Stone WZ, Gray CF, Parvataneni HK, Prieto HA. Clinical evaluation of alpha defensin test following staged treatment of prosthetic joint infections. J Arthroplasty 2019;34:14461451.

63. Okroj KT, Calkins TE, Kayupov E, et al. The alphadefensin test for diagnosing periprosthetic joint infection in the setting of an adverse local tissue reaction secondary to a failed metal-on-metal bearing or corrosion at the head-neck junction. J Arthroplasty 2018;33:1896-1898.

64. Deirmengian CA, Liang L, Rosenberger JP, et al. The leukocyte esterase test strip is a poor rule-out test for periprosthetic joint infection. J Arthroplasty 2018;33:2571-2574.

65. Zagra L, Villa F, Cappelletti L, et al. Can leucocyte esterase replace frozen sections in the intraoperative diagnosis of prosthetic hip infection? Bone Joint J 2019; 101-B: 372-377.

66. Saitoh Y, Setoguchi T, Yoshioka T, et al. Intraoperative evaluation of polymorphonuclear leukocyte during secondstage revision surgery promote overdiagnosis of persistent periprosthetic joint infection. Acta Orthop Traumatol Turc 2018;52:191-195.

67. Strahm C, Zdravkovic V, Egidy C, Jost B. Accuracy of synovial leukocyte and polymorphonuclear cell count in patients with shoulder prosthetic joint infection. J Bone Jt Infect 2018;3:245-248.

68. Blanc P, Bonnet E, Giordano G, et al. The use of labelled leucocyte scintigraphy to evaluate chronic periprosthetic joint infections: A retrospective multicentre study on 168 patients. Eur J Clin Microbiol Infect Dis 2019 Jun 20;38: 1625- 1631.

69. Falstie-Jensen T, Daugaard H, Søballe K, et al. Labeled white blood cell/bone marrow single-photon emission computed tomography with computed tomography fails in diagnosing chronic periprosthetic shoulder joint infection. J Shoulder Elb Surg 2019;28:1040-1048.

70. Schulz BM, Watling JP, Vosseller JT, Strauch RJ. Markedly elevated intra-articular white cell count caused by gout alone. Orthopedics 2014;37:e739-e742.

Address correspondence to: Dr. Dioscaris R. Garcia

Department of Orthopaedic Surgery Rhode Island Hospital 1 Hoppin Street Providence, RI 02903 USA

E-mail: Dioscaris_garcia@Brown.edu 